\title{
Tribological Rehydration and Its Role on Frictional Behavior of PVA/GO Hydrogels for Cartilage Replacement Under Migrating and Stationary Contact Conditions
}

\author{
Yan Shi ${ }^{1,3}$ - Dangsheng Xiong ${ }^{2} \cdot$ Jianliang $\mathrm{Li}^{2} \cdot$ Long Li $^{1} \cdot$ Qibin Liu $^{1} \cdot$ Daniele Dini ${ }^{3}$
}

Received: 14 June 2020 / Accepted: 10 November 2020 / Published online: 19 December 2020

(c) The Author(s) 2020

\begin{abstract}
Graphene oxide (GO) was incorporated into polyvinyl alcohol (PVA) hydrogel to improve its mechanical and tribological performances for potential articular cartilage replacement application. The compressive mechanical properties, creep resistance, and dynamic mechanical properties of PVA/GO hydrogels with varied GO content were studied. The frictional behavior of PVA/GO hydrogels under stationary and migrating contact configurations during reciprocal and unidirectional sliding movements were investigated. The effects of load, sliding speed, diameter of counterface, and counterface materials on the frictional coefficient of PVA/GO hydrogels were discussed. PVA/0.10wt\%GO hydrogel show higher compressive modulus and creep resistance, but moderate friction coefficient. The friction coefficient of PVA/GO hydrogel under stationary and migratory contact configurations greatly depends on interstitial fluid pressurization and tribological rehydration. The friction behavior of PVA/GO hydrogels shows load, speed, and counterface diameter dependence similar to those observed in natural articular cartilage. A low friction coefficient $(\sim 0.03)$ was obtained from PVA/0.10wt $\%$ GO hydrogel natural cartilage counter pair.
\end{abstract}

\section{Graphical Abstract}

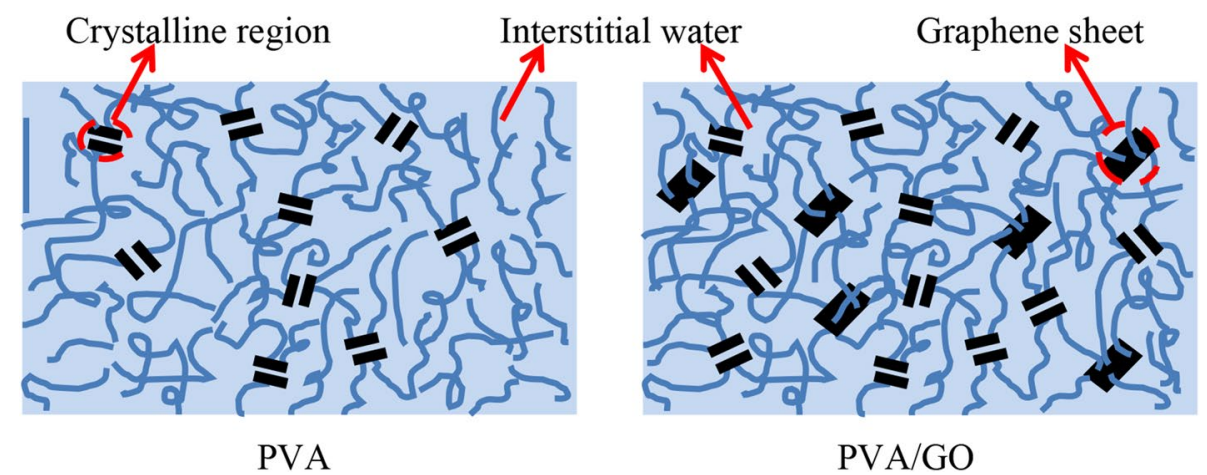

Schematic diagrams of internal structure of PVA and PVA/GO hydrogel

Keywords PVA/GO hydrogel $\cdot$ Porous biphasic structure $\cdot$ Migrating $\cdot$ Stationary $\cdot$ Tribological rehydration

Yan Shi

shiyan1262006@126.com; y.shi@imperial.ac.uk

Guizhou University, Guiyang 550025, China

2 Nanjing University of Science and Technology, Nanjing 210094, China

3 Tribology Group, Department of Mechanical Engineering, Imperial College London, London SW7 2AZ, UK

\section{Introduction}

Hydrogels have been investigated as cartilage alternatives for decades [1-7]. Among hydrogels developed for biomedical applications, polyvinyl alcohol (PVA) hydrogel has attracted great attentions as a potential articular cartilage substitute due to its non-toxicity, high water content, good lubrication 
[8-11]. Nevertheless, pure PVA hydrogel lacks sufficient load-bearing capacity and wear resistance, which hinders its development [12].

Incorporation of graphene oxide (GO) in PVA matrix is considered as one of the effective ways to reinforce hydrogel. The abundant oxygen-containing functional groups on the GO sheets, including the hydroxyl, epoxy, carbonyl, and carboxyl groups, endow GO good water affinity. The good dispersion of GO in water is vital for the nanofiller to function at its best [13-15]. Liang et al. [16] has confirmed the efficient load transfer between the graphene and PVA matrix. As a result, the mechanical properties of the graphene-based nanocomposite with molecule-level dispersion were significantly enhanced. The water-locking and cross-linking effects of GO had been demonstrated in our previous study [17].

The remarkable load-bearing capacity and excellent lubricating ability of the natural cartilage are derived to its porous biphasic structure in which the collagen-proteoglycan network serves as the solid phase and the interstitial fluid within the network acts as the fluid phase. Both phases are incompressible and immiscible, and the fluid phase pressurizes under sliding to share the loads on its solid matrix $[18,19]$. McCutchen [20,21] proposed the weeping lubrication mechanism for the natural cartilage by rubbing against a large glass flat to explain its extraordinary frictional response due to the exudation of interstitial fluid. Moore and Burris [22] confirmed that the pressurized fluid in the inlet of contact that flowed into the cartilage during sliding was responsible for the interstitial fluid recovery and load support for cartilage under stationary contact condition known as tribological rehydration. Murakami et al. [23] elucidated the ingenious lubrication mechanism in natural synovial joints from the view of the adaptive multimode lubrication mechanism. Recently, Porte et al. [24] argued that biphasic lubrication mechanism was not applicable to hydrogels due to their isotropic structure. They proposed that fluid load support was not the main driver behind the tribological behavior of the PVA hydrogels under stationary contact, but a nonreplenished lubricant mechanism could be responsible for the variation in friction response of such hydrogels.

Investigation of the frictional response under various contact configurations is key to design the hydrogel materials for cartilage replacement. Most researchers focused on unidirectional or reciprocating friction of cartilage [25-27], and few researchers have studied the influence of contact modes on friction properties of hydrogels [28, 29]. Schiavinato and Whiteside [30] conducted both migrating and stationary contact tests on bovine cartilage to assess the efficacy of various lubricants. Dunn et al. [28] explored the distinct tribological behaviors of three kinds of interfaces including migrating, stationary, and Gemini (self-mated cartilage) contacts. To better understand the frictional behavior of PVA/ GO hydrogel under the migrating and stationary contacts, the lubrication behaviors could be interpreted from the view of exudation and imbibition of interstitial liquid or tribological rehydration.

Intrinsic property and surface quality of the counterparts are also considerable factors that significantly influence the frictional response. Oungoulian et al. [31] demonstrated that more damage and higher friction coefficient occurred for bovine articular cartilage when sliding against stainless steel than against cobalt chromium alloy or glass. Frictional behaviors of PVA/GO hydrogel under different contact configurations and various movement modes have not been fully evaluated yet.

In this study, PVA/GO composite hydrogels with different amounts of GO were prepared by the repeated freezethawing method. The effects of movement modes, contact configurations, load, sliding speed, diameter of counterface, and the counterface materials on the friction properties of PVA/GO composite hydrogels were investigated. The biphasic lubrication behaviors of PVA/GO under migrating and stationary contact conditions were discussed.

\section{Experimental}

\subsection{Preparation of PVA/GO Composite Hydrogels}

GO was supplied by Nanjing FAME Bearing Co. Ltd., Nanjing, China. PVA $>99 \%$ saponified with a polymerization degree of 1700 was obtained from Kuraray Co. Ltd., Tokyo, Japan. To prepare PVA/GO composite hydrogels, PVA powders were dissolved in deionized water at $95{ }^{\circ} \mathrm{C}$ to form an aqueous solution. GO aqueous suspension with $20 \mathrm{~min}$ of ultrasonic agitation in deionized water was dripped into the PVA solution. These two solutions were mixed to obtain samples with $15 \%$ of polymer concentration and various ratios of GO/PVA $(0,0.05,0.10,0.15$, and $0.20 \%(w / w))$. These mixtures were stirred until the homogenous solutions were created. After being held at a higher temperature to remove air bubbles, the mixtures were poured into molds and subjected to five freezing-thawing cycles; each cycle was performed by freezing at $-20{ }^{\circ} \mathrm{C}$ for $18 \mathrm{~h}$, followed by thawing at room temperature for $6 \mathrm{~h}$.

\subsection{Characterization Techniques}

The morphologies of GO powders and the cross-section morphologies of PVA/GO hydrogels were characterized by Scanning electron microscopy (SEM, FEI Quanta 250FEG, German). The hydrogel samples were placed in freezer dryer (FD-1A-50, China) for at least 2 days to remove water, and then the samples were sputter-coated with a layer of gold for SEM observations. The thicknesses of the GO sheets were 
determined by an FM-Nanoview 1000 atomic force microscope (AFM; Suzhou Flying-man Precision Instrument Co., Ltd., Suzhou, China). Fourier transform infrared ray (FT-IR, Nicolet MAGNA-IR 750, USA) spectroscopy of GO, pure PVA, and PVA/GO composite hydrogel was obtained in the range of 4000-500 $\mathrm{cm}^{-1}$ with the resolution of $4 \mathrm{~cm}^{-1}$. X-ray diffraction (XRD) patterns were obtained with a BrukerAXS D8 Advance X-ray diffractometer (Bruker, Germany) with $\mathrm{Cu} K \alpha$ radiation $\left(\lambda=0.1541 \mathrm{~nm}\right.$ ) from 5 to $60^{\circ}$ (in steps of $0.02^{\circ}$ ). The tube voltage and tube current were kept at $40 \mathrm{kV}$ and $40 \mathrm{~mA}$, respectively. The relative crystallinity degree was calculated as the ratio of the PVA crystalline area to the entire area of the spectrum.

For all the hydrogel samples, the water content, $W$, was determined according to Eq. (1):

$W=\frac{M_{\mathrm{s}}-M_{\mathrm{d}}}{M_{\mathrm{s}}}$

where $M_{\mathrm{s}}$ and $M_{\mathrm{d}}$ represent the fully swollen and completely dried sample, respectively. Five independent samples were tested for each hydrogel $(n=5)$.

\subsection{Mechanical Property Tests}

Unconfined compression tests were conducted on INSTRON5943 at a rate of $4 \mathrm{~mm} \mathrm{~min}^{-1}$ until the compression strain reached $65 \%$. The sample size was approximately $12 \mathrm{~mm}$ in diameter and $4 \mathrm{~mm}$ in height. The compressive tangent modulus of hydrogels at different strain regions was determined by the finite difference method [32]. Three independent samples were tested for each hydrogel $(n=3)$.
The indentation creep tests were conducted on an UMTII multifunctional micro-friction tester in deionized water at room temperature. The hydrogel samples were cut into cylindrical disks with $20 \mathrm{~mm}$ diameter and $4 \mathrm{~mm}$ thickness. A CoCrMo ball with the diameter of $8 \mathrm{~mm}$ was used as the indenter. The samples were placed between the compression plate and the spherical indenter under compression to $5 \mathrm{~N}$, resulting in a nominal contact stress of about $0.47 \mathrm{MPa}$. And then this load was kept constant for 1800s. The displacement and time were recorded. Three indentations at different regions were indented for each hydrogel.

The stress relaxation property was tested on INSTRON5943 by imposing a compression strain of 30\% on hydrogel sample with a diameter of $12 \mathrm{~mm}$ and a height of $4 \mathrm{~mm}$. The loading rate of $4 \mathrm{~mm} \mathrm{~min}^{-1}$ was applied. The normalized relaxation modulus was calculated through Eq. (2) [33]:

$G(t)=\frac{E(t)}{E(0)}=\frac{\sigma(t) / \varepsilon_{0}}{\sigma_{0} / \varepsilon_{0}}=\frac{\sigma(t)}{\sigma_{0}}$

where $E(t)$ and $\sigma(t)$ represent the relaxation modulus and relaxation stress at $t$ (time), respectively. $E(0)$ and $\sigma_{0}$ are the relaxation modulus and relaxation stress at the initial time. $\varepsilon_{0}$ is the constant value of strain ratio.

Dynamic mechanical tests were conducted on a dynamic mechanical thermal analysis instrument (DMTA Q800, USA) in a strain-controlled model. The hydrogel samples were subjected to a sinusoidal shear strain with the strain amplitude of 3\%, which was within the range of linear viscoelastic region, at room temperature. The frequencies were ranging from $0.1 \mathrm{~Hz}$ to $10 \mathrm{~Hz}$. The flat rectangular samples with $25 \mathrm{~mm}$ parallel plates and $4 \mathrm{~mm}$ in thickness were used. The dynamic viscoelastic functions such as storage modulus

Table 1 Friction test conditions

\begin{tabular}{ll}
\hline Factor & Test condition \\
\hline $\begin{array}{l}\text { Movement mode } \\
\text { Contact mode }\end{array}$ & Unidirectional, reciprocal \\
& Migratory contact (a), stationary contact (b) \\
&
\end{tabular}


$\left(G^{\prime}\right)$ and loss modulus $\left(G^{\prime \prime}\right)$ were measured as a function of frequency. Loss factor, $\tan \delta$, was expressed as the following equation:

$\tan \delta=\frac{G^{\prime \prime}}{G^{\prime}}$.

\subsection{Frictional Tests}

The friction properties of the hydrogels were evaluated on a UMT-II multifunctional friction tester at room temperature. Various test conditions are summarized in Table 1. Deionized water was used as liquid lubricant in all tests to form lubricant films on the frictional interface. Otherwise, it also supplied liquid for the rehydration. The friction test duration was $30 \mathrm{~min}$. Before sliding, the friction pairs were kept contact at an applied load for $30 \mathrm{~s}$. Each test condition was performed once on three different samples. The schematic in Table 1 displayed two different contact configurations.
Migrating contact was provided by sliding the flat PVA/GO hydrogel (45 $\mathrm{mm}$ in diameter and $4 \mathrm{~mm}$ in thickness) against CoCrMo ball with various diameters $(8 \mathrm{~mm}$ is the default option unless otherwise stated). For the stationary contact, PVA/GO hydrogel ( $2 \mathrm{~mm}$ in thickness) covered on a stainless steel ball (6 $\mathrm{mm}$ in diameter) was served as the upper sample, and the chosen counterfaces included the CoCrMo (the default counterface unless otherwise specified), Ti alloy, ultra-high molecular weight polyethylene (UHMWPE), and polyetheretherketone (PEEK) disk, and the natural cartilage. The knee joint of the three-month-old New Zealand pig was obtained from a local butcher. Cartilage sample was extracted along the central region of porcine femoral condyle with $\sim 5 \mathrm{~mm}$ in width, and its thickness included $\sim 1 \mathrm{~mm}$ of cartilage attached to $\sim 1.5 \mathrm{~mm}$ of subchondral bone. The cartilage sample was washed to remove debris and then stored in physiological saline at $4{ }^{\circ} \mathrm{C}$ before testing. The relevant parameters of these counterfaces are summarized in Table 2. The time-dependent friction coefficient data was recorded
Table 2 Elements used for various friction counterparts

\begin{tabular}{llll}
\hline Friction counterface & Elastic modulus $(E / \mathrm{MPa})$ & Poisson's ratio $(\nu)$ & Roughness $(R a / \mu \mathrm{m})$ \\
\hline CoCrMo & $241 \times 10^{3}$ & 0.29 & $\sim 0.02$ \\
Ti & $110 \times 10^{3}$ & 0.34 & $\sim 0.06$ \\
UHMWPE & 750 & 0.46 & $0.1-0.2$ \\
PEEK & $4 \times 10^{3}$ & 0.40 & $0.1-0.2$ \\
Cartilage & $0.3-2[34,35]$ & $0.05-0.5[34,36]$ & $\sim 8.5[37]$ \\
\hline
\end{tabular}
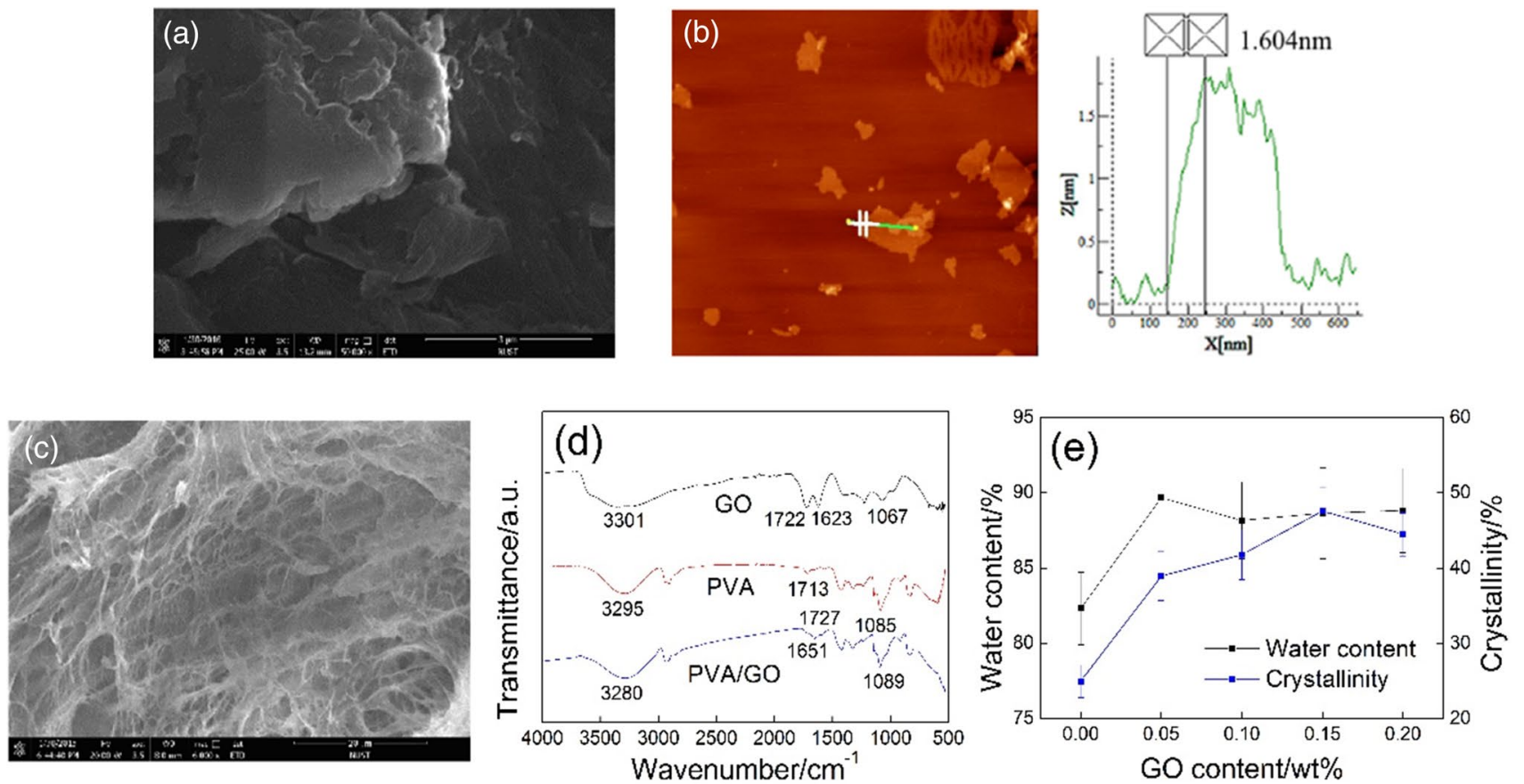

Fig. 1 Characterization results of GO and PVA/GO hydrogel. a SEM and b AFM images of GO; c SEM image and d FT-IR spectra of PVA/0.10wt\%GO hydrogel; e water content and crystallinity of PVA/GO hydrogels 
continuously and the average friction coefficient during the entire test was calculated.

\section{Results}

\subsection{Characterization of GO and PVA/GO Hydrogel}

The GO nanosheet presents the apparent lamellar structure and their average sizes are in the range of $100-300 \mu \mathrm{m}$ (Fig. 1a). The AFM image of GO indicates the results of less aggregation, and its thickness is about $1.48 \mathrm{~nm}$ (Fig. 1b), which is within the thickness range of the single-layer GO sheets [38]. Compared with pure PVA hydrogel [17], PVA/ GO composite hydrogel demonstrates a denser three-dimensional porous network structure (Fig. 1c).

Figure 1d gives the FT-IR spectra of GO, pure PVA and PVA/GO composite hydrogel. The absorption peaks at $3000-3700 \mathrm{~cm}^{-1}$ for both GO and PVA correspond to $\mathrm{O}-\mathrm{H}$ stretching vibration and the absorbed water. It is inevitable that water is absorbed on their surface from the surrounding environment with a humidity of about $50 \%$. The characteristic peaks located at 1722,1623 , and $1067 \mathrm{~cm}^{-1}$ in the spectrum of $\mathrm{GO}$ are attributed to $\mathrm{C}=\mathrm{O}, \mathrm{C}=\mathrm{C}$, and $\mathrm{C}-\mathrm{O}-\mathrm{C}$ stretching vibrations, respectively. For PVA/GO composite hydrogel, the $-\mathrm{OH}$ stretching peak is shifted to a small wavenumber $\left(3280 \mathrm{~cm}^{-1}\right)$ comparing with pure PVA. The oxygen-containing functional groups on the surface of GO form hydrogen bonding with the hydroxyl groups on the PVA chains, which reduces the intermolecular hydrogen bonding between PVA molecular chains. This interfacial interaction between GO and PVA matrix is important for the efficient load transfer in the PVA/GO composite hydrogels and hence for the improvement of anti-deformability and

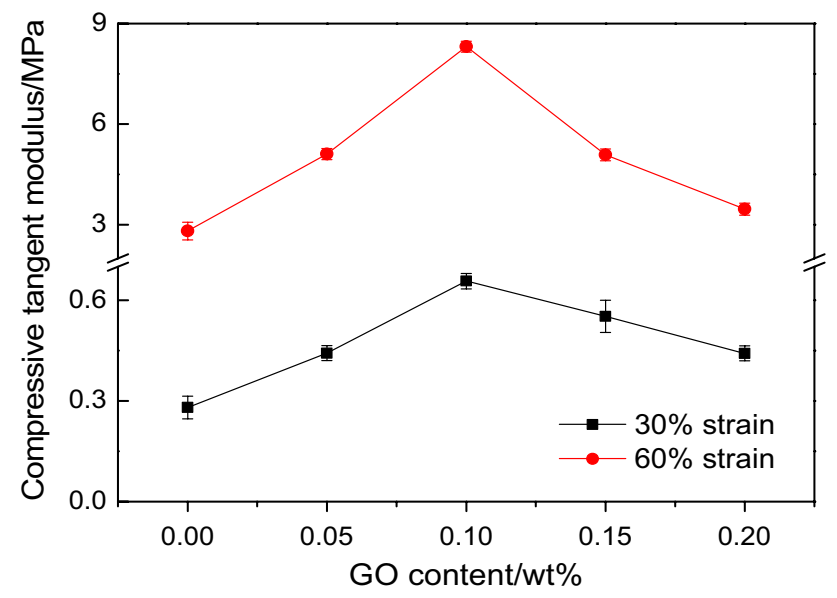

Fig. 2 Unconfined compression tangent modulus of PVA/GO hydrogels

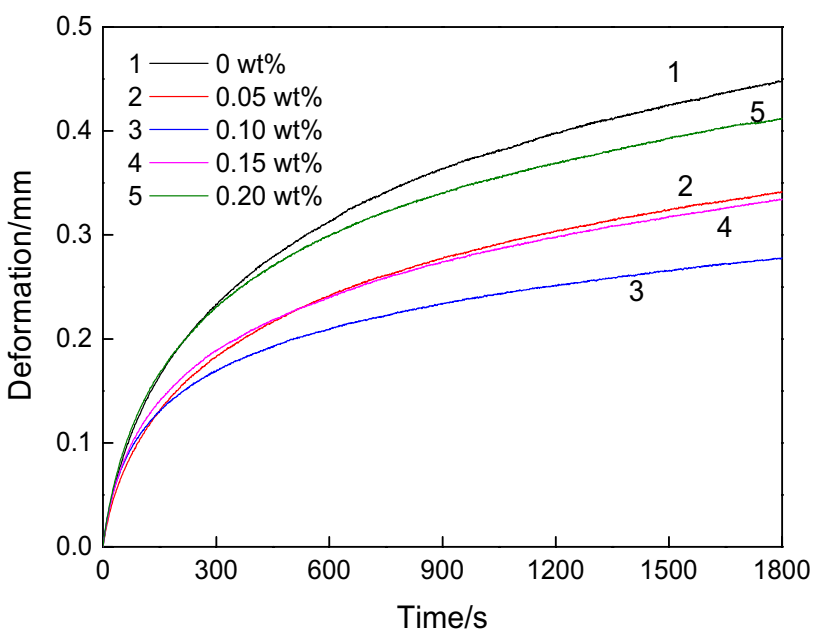

Fig. 3 Indentation creep characteristics of PVA/GO composite hydrogels

wear resistance $[16,17]$. Moreover, those abundant oxygencontaining functional groups on GO sheets and PVA chain are also conducive to trapping water for interstitial fluid pressurization [4].

The crystallinity plays an important role in the mechanical properties of PVA semi-crystalline polymer. The crystallinity of PVA/GO composite hydrogels as a function of GO content is shown in Fig. 1e. It is manifested that the crystallinity of PVA hydrogel significantly increases with the incorporation of GO due to a molecule movement restriction effect [4]. With the further increase of GO content, the crystallinity of PVA/GO composite hydrogels experiences a decreasing tendency because the symmetry and regularity of the polymer chains are broken by the great cross-linking between the GO sheets and PVA matrix.

After the GO is incorporated, water content of PVA/GO composite hydrogels is significantly increased compared with pure PVA hydrogel. Water content of PVA/GO composite hydrogels with various GO contents show similar values, as shown in Fig. 1e.

\subsection{Unconfined Compressive Behaviors of PVA/GO Hydrogel}

Figure 2 shows that the compressive tangent modulus of the PVA hydrogel is significantly enhanced by incorporation of GO. The compressive tangent modulus of PVA/GO composite hydrogels increases first and then decreases with the increase of GO content, and the maximum value is obtained with the addition of $0.10 \mathrm{wt} \% \mathrm{GO}$. At the strain level of $30 \%$, the compressive tangent modulus for the PVA/0.10wt\%GO hydrogel is $0.66 \mathrm{MPa}$, which is improved by about $136 \%$ compared to pure PVA hydrogel. The addition of GO provided a higher affinity for water and a denser porous network 


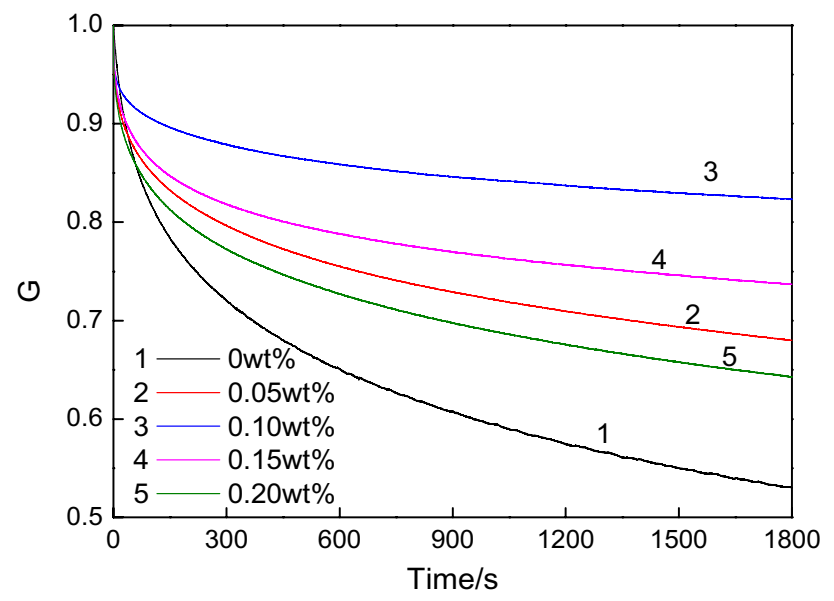

Fig. 4 Normalized relaxation modulus of PVA/GO composite hydrogels as a function of time

for PVA/GO hydrogels. Furthermore, GO impedes the water infiltration between the porous network, which is conducive to the improvement of water-locking ability of the hydrogel.
Therefore, PVA/GO hydrogels with higher amount of GO loading would have a higher load-bearing capacity. However, excess GO loading deteriorated the mechanical properties due to the aggregation of GO fillers [17].

\subsection{Viscoelastic Property of PVA/GO Hydrogel}

The indentation creep properties of PVA/GO hydrogels varied with GO contents are presented in Fig. 3. It indicates that the PVA/GO hydrogels deform fast at the initial stage. Then the deformation increases slowly over time and achieves equilibrium finally. The deformation strain in the creep test dramatically decreases after incorporation of GO. The deformation displacement decreases first and then increases with the increase of GO content, which is consistent with the tendency of compressive tangent modulus as a function of GO content. When the GO content is $0.10 \mathrm{wt} \%$, the minimum creep deformation is obtained, which is $\sim 38 \%$ lower than pure PVA hydrogel.

The normalized compressive relaxation modulus as a function of time for PVA/GO hydrogels with various GO
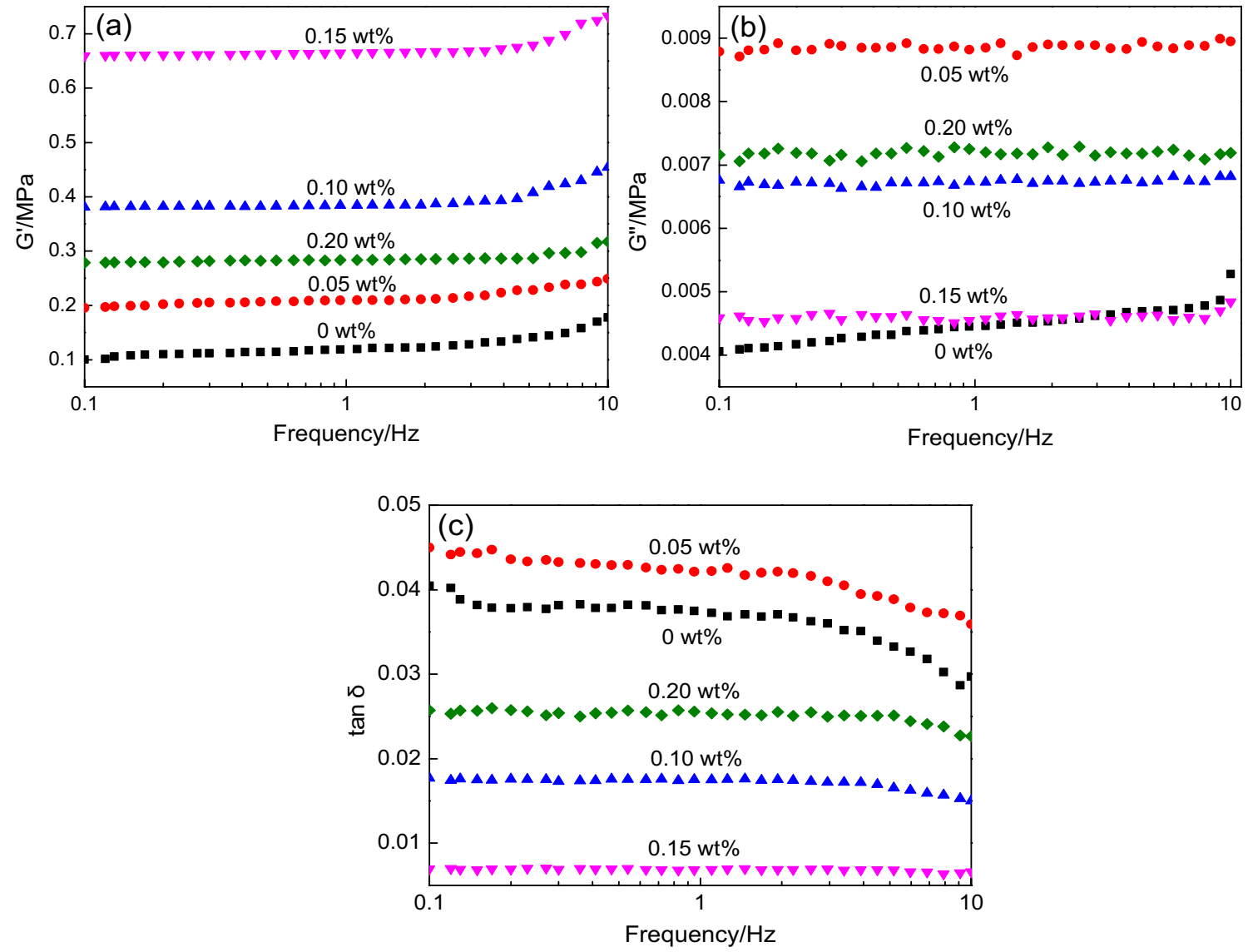

Fig. 5 a Storage modulus, $\mathbf{b}$ loss modulus, and $\mathbf{c}$ loss factor of PVA/GO composite hydrogels as a function of frequency 
Table 3 Average friction coefficient of the PVA/GO hydrogels obtained under various test conditions

\begin{tabular}{lllll}
\hline Test condition & Unidirectional-migratory & Unidirectional-Stationary & Reciprocal-migratory & Reciprocal-stationary \\
\hline PVA & - & $0.183 \pm 0.014$ & $0.296 \pm 0.025$ & $0.291 \pm 0.012$ \\
PVA/0.05wt\%GO & $0.096 \pm 0.007$ & $0.137 \pm 0.010$ & $0.183 \pm 0.009$ & $0.274 \pm 0.017$ \\
PVA/0.10wt\%GO & $0.088 \pm 0.004$ & $0.082 \pm 0.004$ & $0.174 \pm 0.012$ & $0.206 \pm 0.021$ \\
PVA/0.15wt\%GO & $0.079 \pm 0.006$ & $0.060 \pm 0.005$ & $0.108 \pm 0.015$ & $0.260 \pm 0.014$ \\
PVA/0.20wt\%GO & $0.070 \pm 0.004$ & $0.040 \pm 0.006$ & $0.139 \pm 0.020$ & $0.215 \pm 0.018$ \\
\hline
\end{tabular}
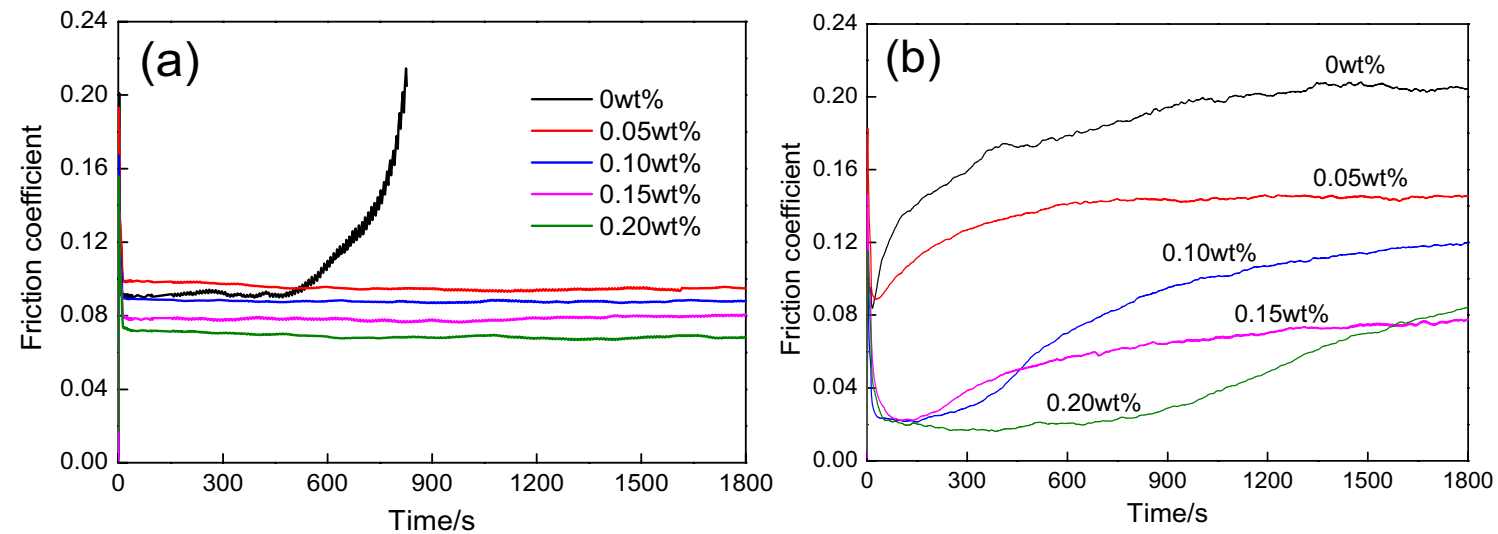

Fig. 6 Friction coefficient of PVA and PVA/GO hydrogel vs. time during unidirectional sliding under various test conditions: a migrating contact; b stationary contact $(5 \mathrm{~N}, 80 \mathrm{~mm} / \mathrm{s})$

content is displayed in Fig. 4. The results demonstrate two typical stages, namely, the rapid and slow relaxation stages [33]. The normalized relaxation modulus of PVA/GO composite hydrogels is higher than that of pure PVA hydrogel, indicating that the incorporation of GO improves the ability of hydrogels to dissipate static loads. An increase in normalized relaxation modulus is obtained with the increase of GO content due to the excellent interfacial bonding between GO sheets and PVA matrix [39]. The highest normalized relaxation modulus is achieved when the GO content is $0.10 \mathrm{wt} \%$. However, the value decreases as the GO content further increases. The aggregation of GO at the excess addition amount results in the decreasing relaxation modulus.

Figure 5 exhibits the evolution of storage modulus, loss modulus, and the loss factor of different PVA/GO hydrogels in the range of test frequencies. Storage modulus of PVA/ GO hydrogels is far higher than loss modulus, indicating the "strong" hydrogel characteristic. A slight increase in storage modulus as a function of frequency is obtained (Fig. 5a), while there is little difference in loss modulus of PVA/GO hydrogels with various amount of GO (Fig. 5b).

As shown in Fig. 5c, all hydrogels are subjected to a gradual decrease in loss factor with increasing frequency. The storage modulus and loss factor of PVA/GO hydrogels increase first and then decrease with the increase of
GO content, and the lowest loss factor (less than 0.01) is obtained from PVA/0.15wt\%GO hydrogel.

\subsection{Frictional Behaviors of PVA/GO Hydrogel}

With the increase of GO content, the average friction coefficients gradually decrease for both migrating and stationary contact conditions during unidirectional sliding, and the decreasing range under stationary contact is higher than that under migrating contact (Table 3). During unidirectional sliding, the friction coefficient of PVA/GO hydrogel under migrating contact condition is relatively stable (Fig. 6a), while the friction coefficient under stationary contact condition increases gradually to reach stable (Fig. 6b). During reciprocating friction, the friction coefficient under the migrating contact configuration increases rapidly at the initiate stage $(<150 \mathrm{~s})$, and then increases slowly with time (Fig. 7a), whereas the friction coefficient under the stationary contact configuration experiences a gradual increase over the test period (Fig. 7b). On the whole, the friction coefficient increases gradually with time under stationary contact, while it is stable under migrating contact. The GO sheets addition reduces the water infiltration, which is conducive to the improvement of liquid lubrication ability during friction. Therefore, PVA/GO hydrogels with higher 

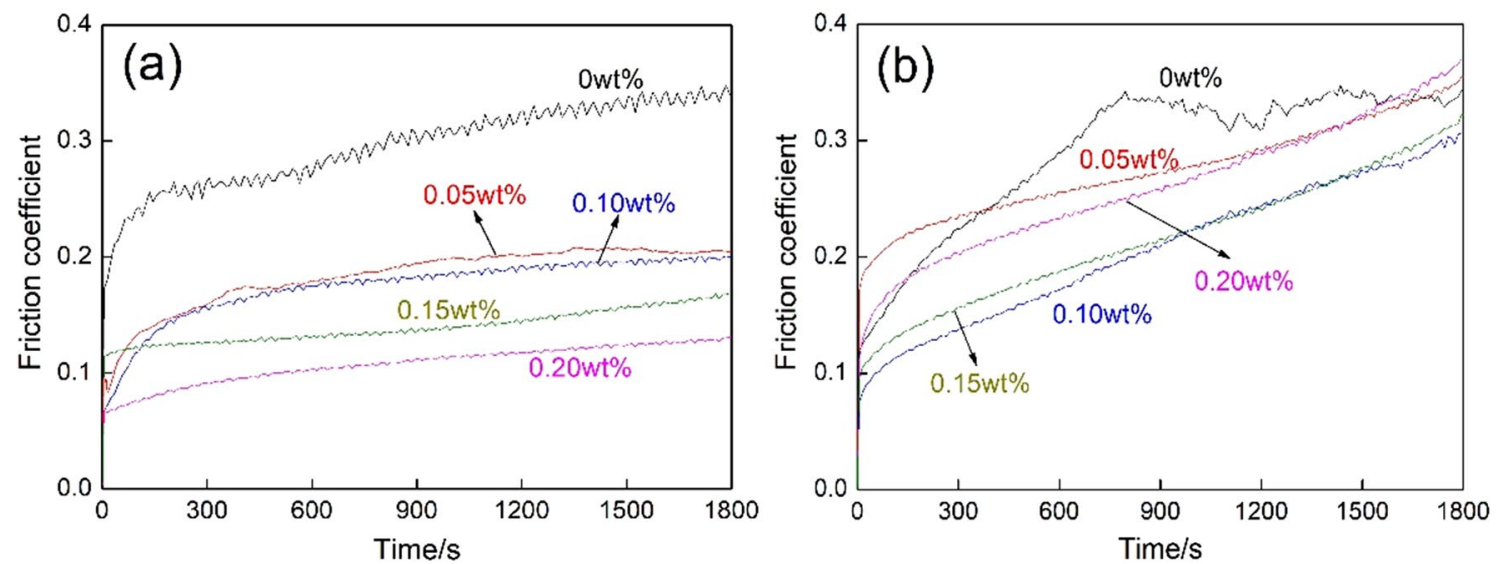

Fig. 7 General trends in reciprocating friction coefficient over time under a migratory contact and b stationary contact (5 N, $8 \mathrm{~mm} / \mathrm{s})$

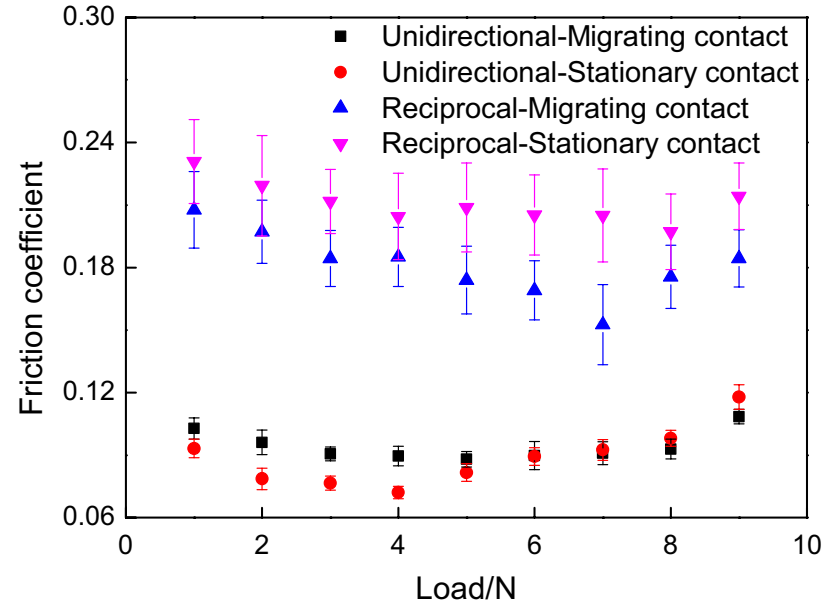

Fig. 8 Effects of load on the friction coefficient of PVA/0.10wt\%GO hydrogels $(80 \mathrm{~mm} / \mathrm{s}$ for unidirectional movement, and $8 \mathrm{~mm} / \mathrm{s}$ for reciprocal movement)

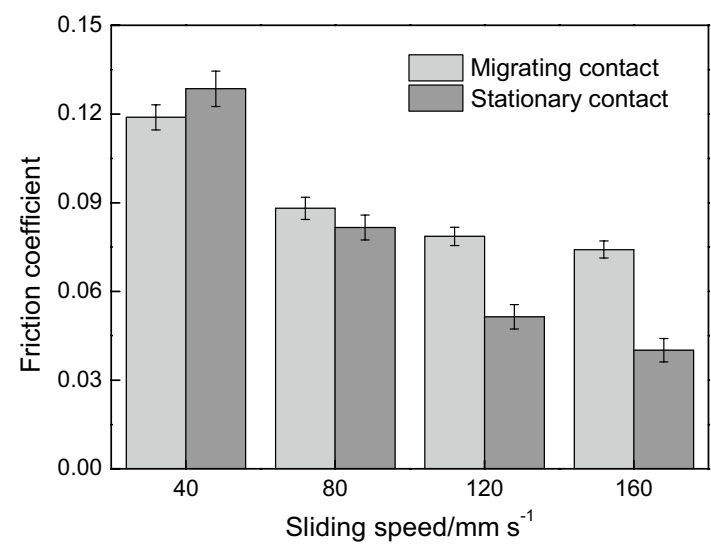

amount of GO loading would produce higher pressurization of interstitial water under stress, which accounts for the decreasing average friction coefficient as a function of GO content.

PVA/GO hydrogels with the GO amount of $0.1 \mathrm{wt} \%$ presents the better compressive properties, which is used for friction properties evaluation. Friction coefficient of PVA/GO hydrogels with $0.1 \mathrm{wt} \% \mathrm{GO}$ as a function of the applied normal load is illustrated in Fig. 8. The friction coefficient decreases first and then increases with increasing load. Figure 9 gives the influence of sliding speed on the average friction coefficient of PVA/GO hydrogel under both migrating and stationary contacts during unidirectional and reciprocal sliding. The friction coefficient decreases with the increase of sliding speed, despite the contact modes. During unidirectional sliding, the friction coefficient under stationary contact experiences a larger

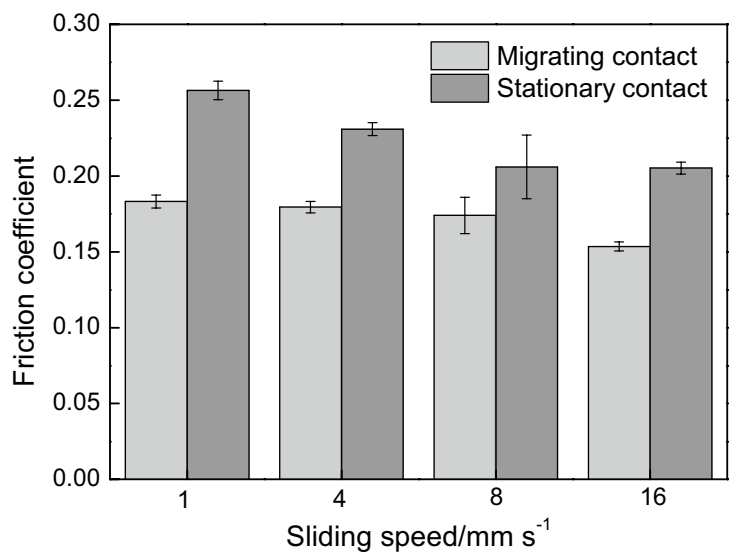

Fig. 9 Effects of sliding speed on the friction coefficient of PVA/0.10wt\%GO hydrogels: a unidirectional sliding and b reciprocal sliding (5 N) 


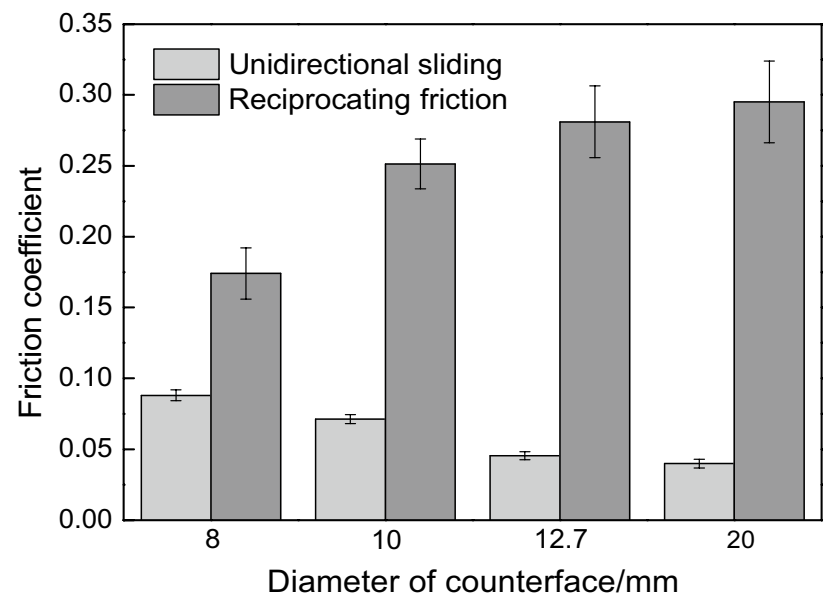

Fig. 10 Effect of diameter of counterface on the friction coefficient of PVA/0.10wt\%GO hydrogels (migratory contact, $5 \mathrm{~N}, 80 \mathrm{~mm} / \mathrm{s}$ for unidirectional movement, and $8 \mathrm{~mm} / \mathrm{s}$ for reciprocal movement)

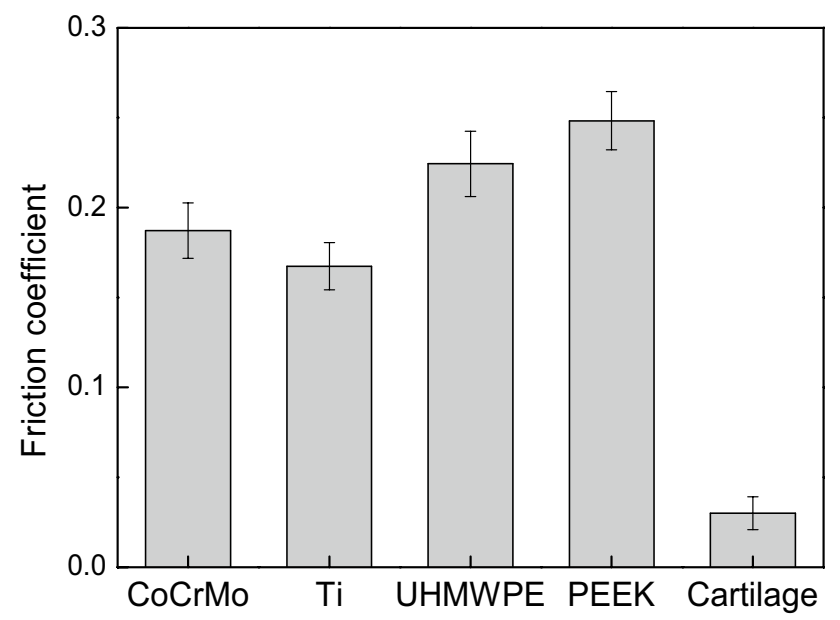

Fig. 11 Effect of counterface material on the friction coefficient of PVA/0.10wt\%GO hydrogels (reciprocal sliding, stationary contact, $5 \mathrm{~N}, 8 \mathrm{~mm} / \mathrm{s}$ )

drop with the increase of sliding speed when compared to that under migrating contact.

Figure 10 shows the effect of the diameter of counterface on the friction coefficient of PVA/GO hydrogel under migrating contacts during unidirectional and reciprocating sliding. The friction coefficient decreases with the increase of diameter of counterface during unidirectional friction. On the contrary, the friction coefficient increases gradually with the diameter of counterface ball during reciprocating friction.

Effect of counterface material on the friction coefficient of PVA/GO hydrogels is shown in Fig. 11. The elastic modulus, Poisson's ratio, and surface roughness of the chosen counterface materials are summarized in Table 2. The
hydrogel-on-CoCrMo and hydrogel-on-Ti contacts exhibit a relatively moderate friction coefficient. The hydrogel-onUHMWPE and hydrogel-on-PEEK contacts show higher friction coefficient. A low friction coefficient $(\sim 0.03)$ is obtained from the hydrogel-on-cartilage contact.

\section{Discussion}

The low permeability (in the range of $10^{-15}$ and $10^{-16} \mathrm{~m}^{4} / \mathrm{Ns}$ ) and a lubricious uppermost layer endow cartilage spectacular load-bearing capacity and super-lubricity [40-42]. PVA/GO hydrogels have similar porous structure as the natural cartilage. However, PVA/GO hydrogels show relatively higher permeability $\left(\sim 10^{-12} \mathrm{~m}^{4} / \mathrm{Ns}[43]\right)$ and absence of lubricious uppermost layer compared with natural cartilage, which leads to different biphasic lubrication behavior compared with the natural cartilage. The water infiltration in PVA/GO hydrogel is partly impeded by GO sheets in some degree as shown in [17]. The biphasic lubrication behaviors of PVA/GO under various movement modes (unidirectional sliding and reciprocal movement) and contact configurations (stationary contact and migratory contact) were discussed and compared with nature cartilage. It is recognized that the friction behaviors of PVA/GO hydrogel under varied movements and contact modes greatly depend on the tribological rehydration, while the biphasic lubrication of hydrogels is guaranteed by the rehydration for interstitial liquid.

\subsection{Biphasic Lubrication Behaviors of PVA/GO Hydrogel Under Various Movement Modes and Contact Configurations}

Ateshian et al. [44] found that cartilage could remain hydration and high level of interstitial fluid pressurization when the migrating rate of contact area on the cartilage surface was faster than the diffusive rate of interstitial fluid within the porous matrix. During the testing under migrating contact, the loss and preservation of interstitial fluid is governed by the Peclet number, $P_{\mathrm{e}}$, defined as:

$P_{\mathrm{e}}=\frac{V \cdot a}{E_{\mathrm{eq}} \cdot k}$

where $V$ is the sliding speed, $a$ is the contact radius, and $E_{\text {eq }}$ and $k$ represent the equilibrium compression modulus and permeability, respectively. The interstitial fluid is retained when $P_{\mathrm{e}}>>1$ or lost with time when $P_{\mathrm{e}}<<1$. The high sliding speed $(40-160 \mathrm{~mm} / \mathrm{s})$ and higher permeability $(k \sim 15.6 \times$ $10^{-12} \mathrm{~m}^{4} / \mathrm{Ns}$ [43]) of hydrogel during unidirectional sliding result in $P_{\mathrm{e}}>>1$.

The PVA/GO hydrogel constantly suffers a constant load in stationary contact during reciprocal sliding with low 
Fig. 12 Lubrication mechanism for $\mathbf{a}$ migrating and $\mathbf{b}$ stationary contacts
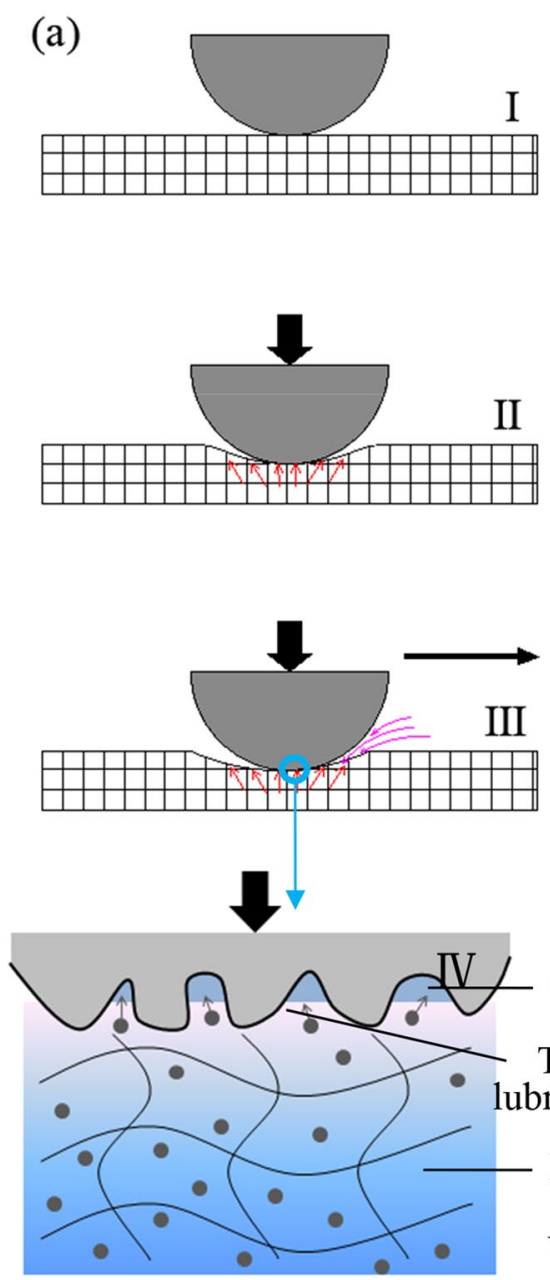

II
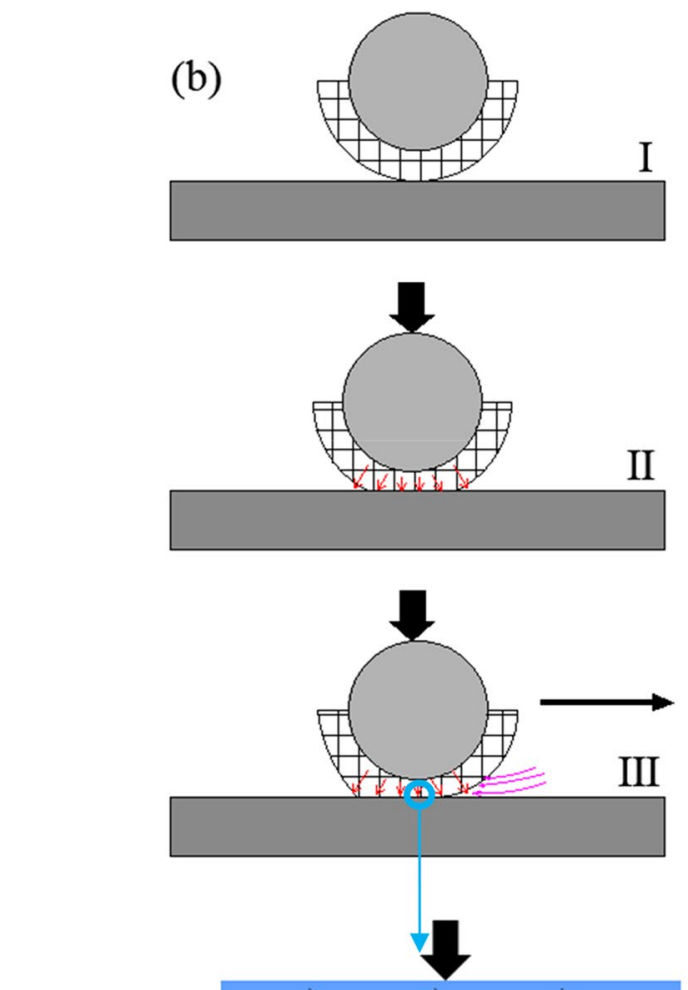

CoCrMo

Trapped

Hydrogel

Water film

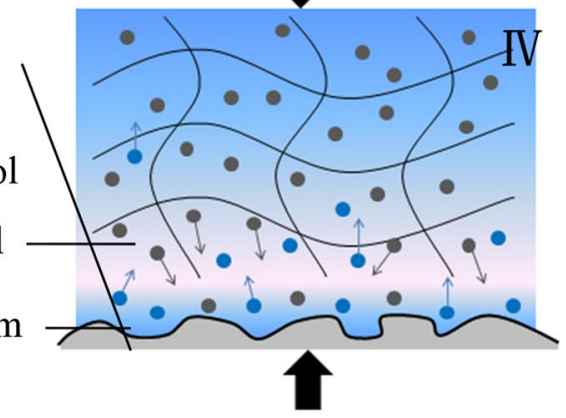

Fig. 13 Imbibition of interstitial liquid for different counter ball diameter

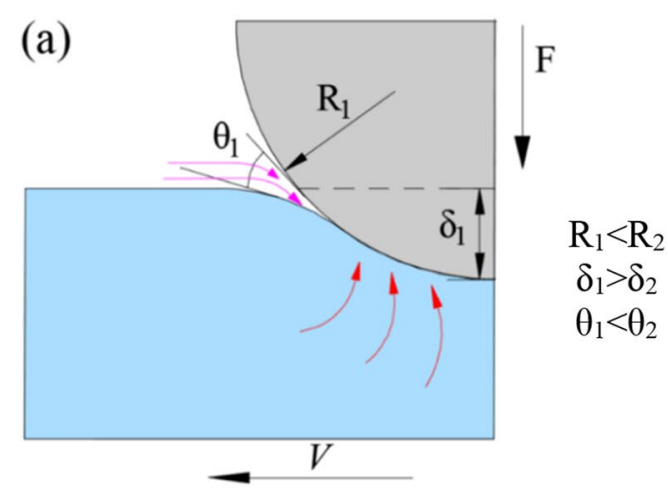

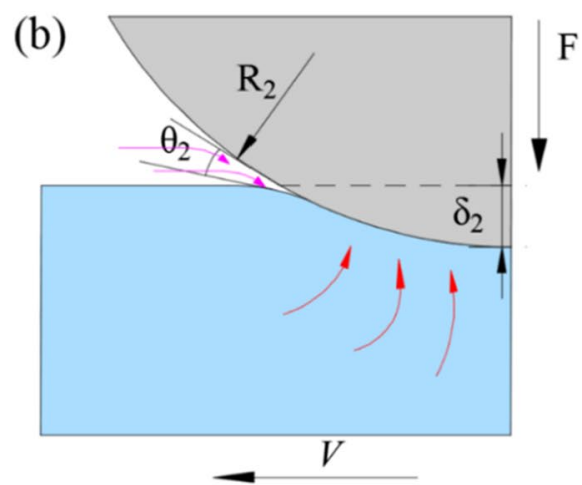

sliding speed (Fig. 12b). The interstitial fluid is lost over time and the rehydration is weak due to constant load, which leads to a larger proportion of solid phase contact. The resulting friction coefficient of PVA/GO hydrogel exhibits strong time dependence due to the loss of interstitial liquid. The cartilage also shows a similar tendency that the friction rises monotonically with increasing reciprocating cycles in stationary contact condition [22, 28]. As reported 
by Murakami [23] and Dunn [28], only a small proportion of load was supported by the liquid film under the stationary contact during reciprocal sliding for cartilage.

Schiavinato and Whiteside [30] conducted both migrating and stationary contact tests on bovine cartilage. Their findings demonstrated that low friction coefficient could be retained over prolonged periods of loading in migrating contact configuration for all kinds of lubricants due to the sustained interstitial fluid pressurization. While the friction coefficient increases monotonically with time until an equilibrium is reached for stationary contact tests due to the subsidence of interstitial fluid pressure.

\subsection{Sliding Speed, Load, Counterface Diameter-Dependent Frictional Behavior}

As demonstrated in Ref. [45] and [46], there is a linear relationship between friction coefficient and the interstitial fluid support. According to Moore and Burris [47], the fluid load fraction, $\mathrm{F}_{\mathrm{f}}$, can be calculated based on the value of $P_{\mathrm{e}}$,

$F_{\mathrm{f}}=\frac{P_{\mathrm{e}}}{P_{\mathrm{e}}+1}$

when Eq. (4) is substituted into Eq. (5), the following expression is obtained,

$F_{\mathrm{f}}=\frac{V \cdot a}{V \cdot a+E_{\mathrm{eq}} \cdot k}$

According to Eq. (4), $P_{\mathrm{e}}$ is proportional to the sliding speed. Increasing the sliding speed in migrating contact yields a higher $P_{\mathrm{e}}$ and $F_{\mathrm{f}}$, thus accompanies with lower friction coefficient (Fig. 9). Dunn et al. [28] demonstrated that the friction coefficient of hydrogels deceased as the sliding speed in both migrating and stationary contacts, and the stronger speed dependence was obtained in migrating contact conditions. The friction coefficient of PVA/GO hydrogel decreases with the increase of sliding speed in migrating contacts.

The increasing applied normal load results in the larger contact radius $(a)$, following by higher fluid load fraction $\left(F_{\mathrm{f}}\right)$. In the low load region (1-5 N), the exudation of fluid phase may be compensated by rehydration, $F_{\mathrm{f}}$ increases with the applied normal load, which accompanies by the decreasing friction coefficient. In the high load region $(5-10 \mathrm{~N})$, the elevated load results in larger amount of exudative interstitial liquid, which would lead to the sudden decrease of the interstitial fluid load fraction $\left(F_{\mathrm{f}}\right)$ and the increase of friction coefficient.

The influences of the counter ball diameter on the friction property of PVA/GO hydrogels could be illustrated from view of fluid load carrying capacity and rehydration.
These two effects are competing and lead to different friction behaviors. The increasing applied normal load results in the larger contact radius, which leads to higher $P_{\mathrm{e}}$ and is benefit for the reduction of friction coefficient. In contrast, from what concerns rehydration, the larger contact radius would reduce the exposure period of hydrogel surface to the water and limit the chance of rehydration, which leads to the increase of friction coefficient. Otherwise, the wedge effect on the friction behavior of PVA/GO hydrogel is considered for different counterface ball diameter. It has been reported that the friction coefficient of cartilage was mainly related to rehydration induced by the wedge effect and the compressing of liquid into the porous surface during sliding $[22,48]$. The larger diameter of counter ball results in lower angle of wedge (Fig. 13). With the increase of counterface ball diameter, the tribological rehydration becomes weaker partly due to the weaker imbibition.

The rehydration is dominant during reciprocal sliding due to the lower sliding speed $(V)$ and the large variation of rehydration linked to the different exposure of the surface to the fluid [46], while the liquid load supporting is relatively stable and play the dominant role for friction coefficient during unidirectional sliding with relatively high speed (higher $\left.P_{\mathrm{e}}\right)$. Therefore, the coefficient of friction increases as the counter ball diameter during reciprocating movement, while the friction coefficient decreases with the counter ball diameter under unidirectional sliding.

In this study, the experiment model can be considered as a soft and thin layer on a rigid body, which is consistent with Shull's report $[49,50]$. Modification to the Hertzian contact theory is necessary when the compliant materials is thin that it cannot be appropriately as an elastic half-space. Shull $[49,50]$ introduced an important parameter, the thickness of the hydrogel $(h)$ relative to the contact radius $(a)$. Therefore, the factor of $(a / h)$ was introduced to modify the values of maximum contact pressure, $P_{\mathrm{H}}$, and contact depth, $\delta_{\mathrm{H}}$, calculated from the Hertzian contact equations. For the model of elastic layer with a thickness of $\mathrm{h}$ on a rigid body, the modification to the load $\left(F^{\prime}\right)$ and contact depth $\left(\delta^{\prime}\right)$ with respect to their Hertzian counterparts can be obtained as follows,

$F^{\prime}=F_{\mathrm{H}}\left(1+\beta\left(\frac{a}{h}\right)^{3}\right)$

$\delta^{\prime}=\delta_{\mathrm{H}}\left(0.4+0.6 \exp \left(\frac{-1.8 a}{h}\right)\right)$

where $\beta$ is equal to 0.15 for low friction (close to a frictionless boundary condition) at the indenter interface [48]. The correction reported in Eq. (7) can be used to derive the apparent increase in stiffness of the hydrogel bonded to the rigid substrate, hence enabling the derivation in first 
Table 4 The calculated and modified values of contact depth, contact radius, and contact pressure for different diameter of CoCrMo ball $(5 \mathrm{~N}$, $80 \mathrm{~mm} / \mathrm{s}$, migrating contact)

\begin{tabular}{lllllll}
\hline $\begin{array}{l}\text { Diameter of ball } \\
(\mathrm{mm})\end{array}$ & $\delta_{\mathrm{H}}(\mathrm{mm})$ & $a_{\mathrm{H}}(\mathrm{mm})$ & $P_{\mathrm{H}}(\mathrm{MPa})$ & $\delta^{\prime}(\mathrm{mm})$ & $a^{\prime}(\mathrm{mm})$ & $P^{\prime}(\mathrm{MPa})$ \\
\hline 8 & 1.255 & 2.241 & 0.475 & 0.602 & 2.121 & 0.540 \\
10 & 1.165 & 2.414 & 0.410 & 0.546 & 2.261 & 0.556 \\
12.7 & 1.076 & 2.614 & 0.349 & 0.492 & 2.417 & 0.576 \\
20 & 0.925 & 3.041 & 0.258 & 0.406 & 2.730 & 0.631 \\
\hline
\end{tabular}

Table 5 The calculated and modified values of contact depth, contact radius, and contact pressure for different friction counterparts $(5 \mathrm{~N}$, $80 \mathrm{~mm} / \mathrm{s}$, stationary contact)

\begin{tabular}{lllllll}
\hline Counterpart material & $\delta_{\mathrm{H}}(\mathrm{mm})$ & $a_{\mathrm{H}}(\mathrm{mm})$ & $P_{\mathrm{H}}(\mathrm{MPa})$ & $\delta^{\prime}(\mathrm{mm})$ & $a^{\prime}(\mathrm{mm})$ & $P^{\prime}(\mathrm{MPa})$ \\
\hline CoCrMo & 1.255 & 2.241 & 0.475 & 0.602 & 2.121 & 0.540 \\
$\mathrm{Ti}$ & 1.255 & 2.241 & 0.475 & 0.602 & 2.121 & 0.540 \\
UHMWPE & 1.256 & 2.242 & 0.475 & 0.603 & 2.122 & 0.540 \\
PEEK & 1.255 & 2.241 & 0.475 & 0.602 & 2.121 & 0.540 \\
Cartilage & 1.992 & 2.823 & 0.299 & 0.891 & 2.574 & 0.379 \\
\hline
\end{tabular}

approximation of the changes in contact radius and maximum contact pressure with respect to the solution originally obtained using Hertzian theory for the hydrogel treated as a semi-infinite body. In the migrating contact configuration, the modified data of contact depth $\left(\delta^{\prime}\right)$, contact radius $\left(a^{\prime}\right)$ and contact pressure $\left(P^{\prime}\right)$ with various diameter of counter ball are listed in Table 4 . The modified values $a^{\prime}, \delta^{\prime}$ and $P^{\prime}$ for different counterpart materials are summarized in Table 5. The $a$ in Eq. (4) and Eq. (6) should be replaced by the value of modified contact radius $\left(a^{\prime}\right)$ as shown in Tables 4 and 5 .

\subsection{Effect of Counterface Materials on Friction Coefficient}

The friction coefficient of PVA/0.10\%GO hydrogel shows little difference when rubbing against $\mathrm{CoCrMo}$ and $\mathrm{Ti}$ alloy. The friction coefficient of PVA/0.1wt\%GO hydrogel against UHMWPE is higher than those contact with CoCrMo and Ti alloy. The adhesive force for the UHMWPE is lower than Ti alloy and CoCrMo due to its hydrophobicity [51-54], but the plowing force is higher due to the higher roughness of UHMWPE $(R a, \sim 0.1-0.2 \mu \mathrm{m})$ compared with CoCrMo and Ti alloy $(R a, \sim 0.02-0.06 \mu \mathrm{m})$. As a result, higher friction coefficient is produced between UHMWPE and hydrogel during sliding. This is supported by the report from Oungoulian and co-workers showing that higher surface roughness of $\mathrm{CoCr}$ alloy led to higher friction coefficient [31]. For the hydrogel-on-cartilage contact configuration, the mesh-confined lubrication mechanism accounts for the friction coefficient under Gemini contact [28]. The lubricious cartilage uppermost layer on the cartilage also contributes to the lower friction coefficient $(\sim 0.03)$ for hydrogel-oncartilage contact.

\section{Conclusions}

The friction properties of PVA/GO hydrogels were investigated under different contact modes (migrating and stationary), contact configurations, load, sliding speed, diameter of counterface, and the counterface materials. PVA/0.10wt\%GO hydrogel show higher compressive modulus and creep resistance, but moderate friction coefficient. The friction coefficient of PVA/GO hydrogel under stationary and migratory contact configurations greatly depended on interstitial fluid pressurization and tribological rehydration. The friction behavior of PVA/GO hydrogels shows load, speed, and counterface diameter dependence similar to those observed in natural articular cartilage. A low friction coefficient $(\sim 0.03)$ was obtained from PVA/0.10wt\%GO hydrogel-on-natural cartilage counter pair.

Acknowledgments This project is supported by the Fundamental Research Project of Guizhou Science and Technology Department (QKHJC[2019]1085 and QKHJC[2020]1Y230), Science and Technology Planning Project of Guizhou (QKHPTRC[2018]5781-16), and Talent Introduction Research Project of Guizhou University (GZU[2017]01).

Open Access This article is licensed under a Creative Commons Attribution 4.0 International License, which permits use, sharing, adaptation, distribution and reproduction in any medium or format, as long as you give appropriate credit to the original author(s) and the source, provide a link to the Creative Commons licence, and indicate if changes were made. The images or other third party material in this article are included in the article's Creative Commons licence, unless indicated otherwise in a credit line to the material. If material is not included in the article's Creative Commons licence and your intended use is not permitted by statutory regulation or exceeds the permitted use, you will need to obtain permission directly from the copyright holder. To view a copy of this licence, visit http://creativecommons .org/licenses/by/4.0/. 


\section{References}

1. Gombert, Y., Simič, R., Roncoroni, F., Dübner, M., Geue, T., Spencer, N.D.: Structuring hydrogel surfaces for tribology. Adv. Mater. Interfaces 6, 1901320 (2019)

2. Lin, P., Zhang, R., Wang, X., Cai, M., Yang, J., Yu, B., Zhou, F.: Articular cartilage inspired bilayer tough hydrogel prepared by interfacial modulated polymerization showing excellent combination of high load-bearing and low friction performance. ACS Macro Lett. 5, 1191-1195 (2016)

3. Gong, J.P., Katsuyama, Y., Kurokawa, T., Osada, Y.: Double-network hydrogels with extremely high mechanical strength. Adv. Mater. 15, 1155-1158 (2003)

4. Bao, C., Guo, Y., Song, L., Hu, Y.: Poly(vinyl alcohol) nanocomposites based on graphene and graphite oxide: a comparative investigation of property and mechanism. J. Mater. Chem. 21, 13942-13950 (2011)

5. Kumar, B.Y.S., Isloor, A.M., Kumar, G.C.M., Inamuddin, Asiri, A.M.: Nanohydroxyapatite reinforced chitosan composite hydrogel with tunable mechanical and biological properties for cartilage regeneration. Sci. Rep. 9, 15957 (2019)

6. Chen, L., Yin, Y., Liu, Y., Lin, L., Liu, M.: Design and fabrication of functional hydrogels through interfacial engineering. Chin. J. Polym. Sci. 35, 1181-1193 (2017)

7. Zhang, X., Wang, J., Jin, H., Wang, S., Song, W.: Bioinspired supramolecular lubricating hydrogel induced by shear force. J. Am. Chem. Soc. 140, 3186-3189 (2018)

8. Bray, J.C., Merrill, E.W.: Poly(vinyl alcohol) hydrogels for synthetic articular cartilage material. J. Biomed. Mater. Res. 7, 431443 (1973)

9. Bodugoz-Senturk, H., Macias, C.E., Kung, J.H., Muratoglu, O.K.: Poly(vinyl alcohol)-acrylamide hydrogels as load-bearing cartilage substitute. Biomaterials 30, 589-596 (2009)

10. Yarimitsu, S., Yoshida, A., Sasaki, S., Murakami, T., Suzuki, A.: Evaluation of lubrication property of poly(vinyl alcohol) hybrid gel for artificial articular cartilage. Tribol. Online 11, 360-365 (2016)

11. Chen, K., Yang, X., Zhang, D., Xu, L., Zhang, X., Wang, Q.: Biotribology behavior and fluid load support of PVA/HA composite hydrogel as artificial cartilage. Wear 376-377, 329-336 (2017)

12. Baker, M.I., Walsh, S.P., Schwartz, Z., Boyan, B.D.: A review of polyvinyl alcohol and its uses in cartilage and orthopedic applications. J. Biomed. Mater. Res. B. 100, 1451-1457 (2012)

13. Xu, Y., Hong, W., Bai, H., Li, C., Shi, G.: Strong and ductile poly(vinyl alcohol)/graphene oxide composite films with a layered structure. Carbon 47, 3538-3543 (2009)

14. Stankovich, S., Dikin, D.A., Piner, R.D., Kohlhaas, K.A., Kleinhammes, A., Jia, Y., Wu, Y., Nguyen, S.T., Ruoff, R.S.: Synthesis of graphene-based nanosheets via chemical reduction of exfoliated graphite oxide. Carbon 45, 1558-1565 (2007)

15. Si, Y., Samulski, E.T.: Synthesis of Water Soluble Graphene. Nano Lett. 8, 1679-1682 (2008)

16. Liang, J., Huang, Y., Zhang, L., Wang, Y., Ma, Y., Guo, T., Chen, Y.: Molecular-level dispersion of graphene into poly(vinyl alcohol) and effective reinforcement of their nanocomposites. Adv. Funct. Mater. 19, 2297-2302 (2009)

17. Shi, Y., Xiong, D., Li, J., Wang, N.: The water-locking and crosslinking effects of graphene oxide on the load-bearing capacity of poly(vinyl alcohol) hydrogel. RSC Adv. 6, 82467-82477 (2016)

18. Ateshian, G.A., Lai, W.M., Zhu, W.B., Mow, V.C.: An asymptotic solution for the contact of two biphasic cartilage layers. J. Biomech. 27, 1347-1360 (1994)
19. Mow, V.C., Kuei, S.C., Lai, W.M., Armstrong, C.G.: Biphasic creep and stress relaxation of articular cartilage in compression: theory and experiments. J. Biomech. Eng. 102, 73-84 (1980)

20. McCutchen, C.W.: The frictional properties of animal joints. Wear 5, 1-17 (1962)

21. McCutchen, C.W.: Mechanism of animal joints: sponge-hydrostatic and weeping bearings. Nature 184, 1284-1285 (1959)

22. Moore, A.C., Burris, D.L.: Tribological rehydration of cartilage and its potential role in preserving joint health. Osteoarthr Cartilage 25, 99-107 (2017)

23. Murakami, T., Yarimitsu, S., Sakai, N., Nakashima, K., Yamaguchi, T., Sawae, Y.: Importance of adaptive multimode lubrication mechanism in natural synovial joints. Tribol. Int. 113, 306-315 (2017)

24. Porte, E., Cann, P., Masen, M.: Fluid load support does not explain tribological performance of PVA hydrogels. J. Mech. Behav. Biomed. 90, 284-294 (2019)

25. Lopes, J.L., Machado, J.M., Castanheira, L., Granja, P.L., Gama, F.M., Dourado, F., Gomes, J.R.: Friction and wear behaviour of bacterial cellulose against articular cartilage. Wear 271, 2328$2333(2011)$

26. Shi, Y., Xiong, D.: Microstructure and friction properties of PVA/ PVP hydrogels for articular cartilage repair as function of polymerization degree and polymer concentration. Wear 305, 280-285 (2013)

27. Chen, K., Liu, J., Yang, X., Zhang, D.K.: Preparation, optimization and property of PVA-HA/PAA composite hydrogel. Mater. Sci. Eng. C 78, 520-529 (2017)

28. Dunn, A.C., Sawyer, W.G., Angelini, T.E.: Gemini interfaces in aqueous lubrication with hydrogels. Tribol. Lett. 54, 59-66 (2014)

29. Shi, Y., Xiong, D., Zhang, J.: Effect of irradiation dose on mechanical and biotribological properties of PVA/PVP hydrogels as articular cartilage. Tribol. Int. 78, 60-67 (2014)

30. Schiavinato, A., Whiteside, R.A.: Effective lubrication of articular cartilage by an amphiphilic hyaluronic acid derivative. Clin. Biomech. 27, 515-519 (2012)

31. Oungoulian, S.R., Durney, K.M., Jones, B.K., Ahmad, C.S., Hung, C.T., Ateshian, G.A.: Wear and damage of articular cartilage with friction against orthopedic implant materials. J. Biomech. 48, 1957-1964 (2015)

32. Shi, Y., Xiong, D.S., Liu, Y.T., Wang, N., Zhao, X.D.: Swelling, mechanical and friction properties of PVA/PVP hydrogels after swelling in osmotic pressure solution. Mater. Sci. Eng. C 65, 172-180 (2016)

33. Pan, Y.S., Xiong, D.S.: Stress relaxation behavior of nanohydroxyapatite reinforced poly(vinyl alcohol) gel composites as biomaterial. J. Mater. Sci. 45, 5495-5501 (2010)

34. Laasanen, M.S., Töyräs, J., Korhonen, R.K., Rieppo, J., Saarakkalaa, S., Nieminen, M.T., Hirvonen, J., Jurvelin, J.S.: Biomechanical properties of knee articular cartilage. Biorheology $\mathbf{4 0}$, 133-140 (2003)

35. Crockett, R., Roos, S., Rossbach, P., Dora, C., Born, W., Troxler, $\mathrm{H}$.: Imaging of the surface of human and bovine articular cartilage with ESEM and AFM. Tribol. Lett. 19, 311-317 (2005)

36. Wang, C.C.-B., Deng, J.M., Ateshian, G.A., Hung, C.T.: An automated approach for direct measurement of two-dimensional strain distributions within articular cartilage under unconfined compression. J. Biomech. Eng. 124, 557-567 (2002)

37. Saarakkala, S., Wang, S.Z., Huang, Y.P., Zheng, Y.P.: Quantification of the optical surface reflection and surface roughness of articular cartilage using optical coherence tomography. Phys. Med. Biol. 54, 6837-6852 (2009)

38. Chen, L., Xu, Z., Li, J., Li, Y., Shan, M., Wang, C., Wang, Z., Guo, Q., Liu, L., Chen, G., Qian, X.: A facile strategy to prepare functionalized graphene via intercalation, grafting and self-exfoliation of graphite oxide. J. Mater. Chem. 22, 13460 (2012) 
39. Sharma, S.K., Prakash, J., Pujari, P.K.: Effects of the molecular level dispersion of graphene oxide on the free volume characteristics of poly(vinyl alcohol) and its impact on the thermal and mechanical properties of their nanocomposites. Phys. Chem. Chem. Phys. 17, 29201-29209 (2015)

40. Murakami, T., Sakai, N., Yamaguchi, T., Yarimitsu, S., Nakashima, K., Sawae, Y., Suzuki, A.: Evaluation of a superior lubrication mechanism with biphasic hydrogels for artificial cartilage. Tribol. Int. 89, 19-26 (2015)

41. Vikingsson, L., Claessens, B., Gomez-Tejedor, J.A., Gallego Ferrer, G., Gomez Ribelles, J.L.: Relationship between micro-porosity, water permeability and mechanical behavior in scaffolds for cartilage engineering. J. Mech. Behav. Biomed. Mater. 48, 60-69 (2015)

42. Mow, V.C., Lai, W.M.: Recent developments in synovial joint biomechanics. SIAM Rev. 22, 275-317 (1980)

43. Ng, K.W., Torzilli, P.A., Warren, R.F., Maher, S.A.: Characterization of a macroporous polyvinyl alcohol scaffold for the repair of focal articular cartilage defects. J. Tissue Eng. Regen. Med. 8, 164-168 (2014)

44. Ateshian, G.A., Wang, H.Q.: A theoretical solution for the frictionless rolling contact of cylindrical biphasic articular cartilage layers. J. Biomech. 28, 1341-1355 (1995)

45. Krishnan, R., Kopacz, M., Ateshian, G.A.: Experimental verification of the role of interstitial fluid pressurization in cartilage lubrication. J. Orthop. Res. 22, 565-570 (2004)

46. Accardi, M.A., Dini, D., Cann, P.M.: Experimental and numerical investigation of the behaviour of articular cartilage under shear loading-interstitial fluid pressurisation and lubrication mechanisms. Tribol. Int. 44, 565-578 (2011)

47. Moore, A.C., Burris, D.L.: An analytical model to predict interstitial lubrication of cartilage in migrating contact areas. J. Biomech. 47, 148-153 (2014)
48. Burris, D.L., Ramsey, L., Graham, B.T., Price, C., Moore, A.C.: How sliding and hydrodynamics contribute to articular cartilage fluid and lubrication recovery. Tribol. Lett. 67, 46 (2019)

49. Shull, K.R.: Contact mechanics and the adhesion of soft solids. Mater. Sci. Eng. R 36, 1-45 (2002)

50. Shull, K.R., Ahn, D., Chen, W.L., Flanigan, C.M., Crosby, A.J.: Axisymmetric adhesion tests of soft materials. Macromol. Chem. Phys. 199, 489-511 (1998)

51. Xia, Y., Dong, H., Zhang, R., Wang, Y., Hao, X., Li, P., Dong, C.: Interfacial microstructure and shear strength of Ti6Al4V alloy/316L stainless steel joint brazed with Ti333Zr167Cu50xNix amorphous filler metals. Mater. Design 187, 108380 (2020)

52. Kan, Y., Cvjetinovic, J., Statnik, E.S., Ryazantsev, S.V., Anisimova, N.Y., Kiselevskiy, M.V., Salimon, A.I., Maksimkin, A.V., Korsunsky, A.M.: The fabrication and characterization of bioengineered ultra-high molecular weight polyethylene-collagen-hap hybrid bone-cartilage patch. Mater. Today Commun. 24, 101052 (2020)

53. Wang, H., Xu, L., Hu, J., Wang, M., Wu, G.: Radiation-induced oxidation of ultra-high molecular weight polyethylene (UHMWPE) powder by gamma rays and electron beams: a clear dependence of dose rate. Radiat. Phys. Chem. 115, 88-96 (2015)

54. Guo, Z., Pang, X., Yan, Y., Gao, K., Volinsky, A.A., Zhang, T.-Y.: CoCrMo alloy for orthopedic implant application enhanced corrosion and tribocorrosion properties by nitrogen ion implantation. Appl. Surf. Sci. 347, 23-34 (2015)

Publisher's Note Springer Nature remains neutral with regard to jurisdictional claims in published maps and institutional affiliations. 